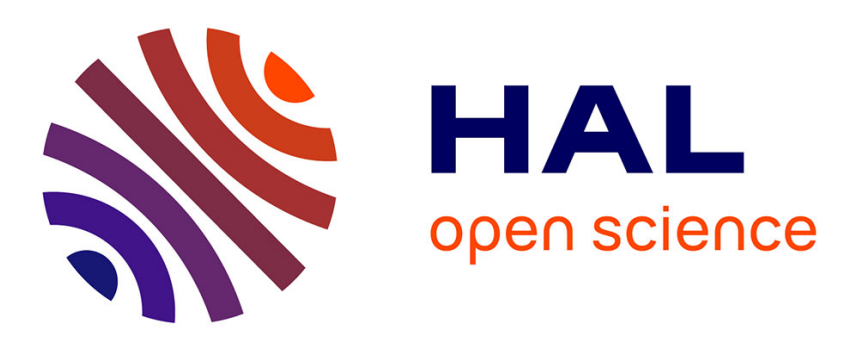

\title{
L'implication des élèves dans la vie de l'établissement : regards croisés des enseignants et des conseillers principaux d'éducation \\ Sylvie Condette
}

\section{- To cite this version:}

Sylvie Condette. L'implication des élèves dans la vie de l'établissement : regards croisés des enseignants et des conseillers principaux d'éducation. Carrefours de l'éducation, 2009, 28 (2), p. 53-64. 10.3917/cdle.028.0053 . hal-01257724

\section{HAL Id: hal-01257724 \\ https://hal.univ-lille.fr/hal-01257724}

Submitted on 18 Jan 2016

HAL is a multi-disciplinary open access archive for the deposit and dissemination of scientific research documents, whether they are published or not. The documents may come from teaching and research institutions in France or abroad, or from public or private research centers.
L'archive ouverte pluridisciplinaire HAL, est destinée au dépôt et à la diffusion de documents scientifiques de niveau recherche, publiés ou non, émanant des établissements d'enseignement et de recherche français ou étrangers, des laboratoires publics ou privés. 


\title{
L'IMPLICATION DES ÉLÈVES DANS LA VIE DE L'ÉTABLISSEMENT : REGARDS CROISÉS DES ENSEIGNANTS ET DES CONSEILLERS PRINCIPAUX D'ÉDUCATION
}

\author{
Sylvie Condette-Castelain
}

Armand Colin | Carrefours de l'éducation

$2009 / 2-n^{\circ} 28$
pages 53 à 64

ISSN 1262-3490

Article disponible en ligne à l'adresse:

http://www.cairn.info/revue-carrefours-de-l-education-2009-2-page-53.htm

Pour citer cet article :

Condette-Castelain Sylvie, «L'implication des élèves dans la vie de l'établissement : regards croisés des enseignants et des conseillers principaux d'éducation »,

Carrefours de l'éducation, 2009/2 n²8, p. 53-64. DOI : 10.3917/cdle.028.0053

Distribution électronique Cairn.info pour Armand Colin.

(c) Armand Colin. Tous droits réservés pour tous pays.

La reproduction ou représentation de cet article, notamment par photocopie, n'est autorisée que dans les limites des conditions générales d'utilisation du site ou, le cas échéant, des conditions générales de la licence souscrite par votre établissement. Toute autre reproduction ou représentation, en tout ou partie, sous quelque forme et de quelque manière que ce soit, est interdite sauf accord préalable et écrit de l'éditeur, en dehors des cas prévus par la législation en vigueur en France. II est précisé que son stockage dans une base de données est également interdit. 


\title{
L'implication des élèves dans la vie de l'établissement: regards croisés des enseignants et des conseillers principaux d'éducation
}

\author{
A sylvie Condette-Castelain \\ sylvie.condette@wanadoo.fr
}

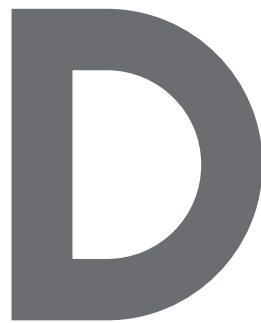

epuis le début des années 1990, les lycéens français ont acquis des droits visant à faciliter leur participation à la vie de l'établissement. Leur rôle au conseil de classe et au conseil d'administration a été réaffirmé et de nouvelles instances participatives ont été créées (conseil de vie lycéenne, assemblée générale des délégués, comité d'éducation à la santé et à la citoyenneté...). La vie scolaire des établissements s'articule ainsi autour des activités organisées pendant le temps passé en classe et celles qui se déroulent en dehors des heures de cours, avec en principe une continuité des apprentissages dans ces différents espaces éducatifs. Si l'on constate que tous les élèves ne s'emparent pas des possibilités offertes, une minorité fait pourtant le choix de s'impliquer au quotidien, en particulier dans les instances consultatives ou délibératives qui orientent, en partie, les décisions politiques de l'établissement. Comment cette « minorité active » est-elle perçue par les personnels de l'établissement? Ceux-ci partagent-ils une conception assez unifiée, homogène de la participation des élèves? Ou au contraire, le statut, la formation, la pratique professionnelle sont-ils autant de variables qui interviennent dans la conception que ces adultes se font de l'implication possible des élèves?

Pour répondre à ces questions, nous reprendrons une partie des résultats d'une recherche centrée sur la participation des lycéens, commencée en 1999 et qui se poursuit actuellement en s'élargissant à d'autres contextes et groupes scolaires. Notre analyse prendra également appui, à partir d'une série d'entretiens, sur la pratique professionnelle d'enseignants, de chefs d'établissement et de conseillers principaux d'éducation.

Nous constaterons tout d'abord que la participation des élèves n'est pas unanimement accueillie dans les lycées qui affirment pourtant valoriser la 
citoyenneté, et qu'elle fait l'objet de divergences, de tensions entre les personnels. Les conseillers principaux d'éducation (CPE) ont plutôt tendance à encourager les initiatives des élèves, même si celles-ci se heurtent aux réticences, voire aux résistances des autres adultes. Nous verrons alors qu'existe un lien étroit entre les missions du métier de CPE telles qu'elles se déclinent officiellement et se mettent en pratique, et la facilitation du processus de responsabilisation des élèves dans l'établissement scolaire.

\section{La participation des élèves: un sujet de controverse au sein de l'établissement scolaire}

La reconnaissance de la parole des élèves et des parents, leur possible participation à la vie de l'établissement, officialisées dès novembre $1968^{1}$, ont modifié en profondeur l'organisation scolaire car il s'agissait pour la première fois d'associer les bénéficiaires du système scolaire à la prise de décisions. Cette ouverture aux « usagers » s'est heurtée à de nombreuses oppositions de la part des personnels qui ne souhaitaient pas, pour un grand nombre, expliquer, clarifier le fonctionnement de l'école et informer les élèves et les familles des choix réalisés, notamment en matière pédagogique. Inégalement mise en œuvre, la participation des élèves et des parents s'est vue institutionnellement limitée et a progressivement perdu son enthousiasme et sa force, tombant parfois même quelque peu dans l'oubli, avant d'être réaffirmée en 1989 dans une loi d'orientation sur l'éducation² qui accorde à l'élève une place centrale dans le système éducatif. À partir de là, de nombreux textes officiels vont promouvoir l'implication des élèves et favoriser leurs initiatives, à la fois en classe mais aussi en dehors.

\section{Des obligations mais aussi des droits}

C'est le décret du 18 février 1991 qui octroie aux élèves, en particulier aux lycéens, de nouveaux droits, assortis d'obligations ${ }^{3}$. Si ces dernières ont permis aux équipes d'établissement de redéfinir, de réactualiser un certain nombre de règles inhérentes à la scolarité de chaque élève, inhérentes également à la vie collective, en rénovant en particulier le règlement intérieur, les droits accordés ont suscité des réactions, parfois vives, de la part des personnels, notamment de certains chefs d'établissement et d'enseignants qui ont vu, dans ce texte, une possible atteinte à l'exercice de leur autorité. Ces droits restent pourtant assez encadrés, dans la mesure où l'on se situe dans un établissement scolaire et donc

\footnotetext{
1. Décret n 68-968 du 8 novembre 1968 .

2. Loi nº 89-486 du 10 juillet 1989 d'orientation sur l'éducation.

3. Décret n 91-173 du 18 février 1991 relatif aux droits et obligations des élèves dans les établissements publics locaux d'enseignement du second degré.
} 
sous l'autorité et la responsabilité de son chef d'établissement, et que la plupart des élèves sont mineurs.

Les lycéens disposent en effet d'un droit d'expression individuelle et collective, mais il ne s'exerce pas de n'importe quelle manière: tout élève a le droit d'exprimer son opinion à l'intérieur de l'établissement scolaire, à condition qu'il en use dans un esprit de tolérance et de respect d'autrui.

Une association composée d'élèves, réunissant éventuellement d'autres membres de la communauté éducative de l'établissement, est autorisée à fonctionner à l'intérieur d'un lycée, après le dépôt préalable d'une copie des statuts de l'association, si le conseil d'administration a donné son accord, et sous réserve que l'objet et l'activité de l'association soient compatibles avec les principes du service public de l'enseignement (en particulier, elle ne peut avoir un objet ou une activité à caractère politique ou religieux). Les lycéens ont un droit de réunion dont les modalités d'exercice sont précisées dans le règlement intérieur de l'établissement.

Le droit de publication est également accordé aux lycéens, dans le respect des règles correspondant à la déontologie de la presse. En effet, même à l'intérieur d'un lycée, les élèves sont soumis à des règles strictes et ne peuvent s'autoriser à tout écrire et tout diffuser, leur responsabilité individuelle pouvant être mise en cause.

Deux types de publication sont possibles: les publications de presse, se référant à la loi du 29 juillet 1881, présentent de lourdes contraintes, comme la désignation d'un directeur de publication qui doit être majeur, la déclaration du titre du journal et de son mode de publication auprès du Procureur de la République, et le dépôt légal de deux exemplaires à chaque publication.

Le deuxième type de publication n'entre pas dans le cadre de la loi de 1881 et ne concerne que les publications diffusées à l'intérieur de l'établissement. Dans ce cas, les lycéens peuvent être mineurs mais ils doivent indiquer au chef d'établissement le nom d'un responsable; ils restent bien évidemment soumis à un certain nombre de règles (les écrits ne doivent pas être diffamatoires, etc.), doivent respecter les principes de neutralité et de pluralisme, et ne pas porter atteinte aux droits d'autrui.

Les droits des élèves, en particulier des lycéens, trouvent leur application et leur concrétisation dans des instances officielles qui leur permettent de s'exprimer. Le conseil de classe organisé chaque trimestre, le conseil de la vie lycéenne (CVL) réunissant à parité dix élèves et dix adultes et consulté avant chaque conseil d'administration, le conseil d'administration dans lequel les élèves délégués ont voix délibérative, sont autant d'espaces de participation offerts aux élèves dans l'établissement scolaire.

Les possibilités d'expression existent donc bien mais on voit nettement que les droits accordés restent sous le contrôle vigilant des adultes de l'établissement, ce qui permet en principe de prévenir, d'éviter les éventuelles difficultés ou dérives. 


\section{Les formes de participation encouragées ou simplement tolérées}

Lorsque l'on interroge les lycéens sur ce que signifie la participation, ils font en tout premier lieu et très majoritairement référence à la participation orale pendant les cours. Celle-ci fait partie des obligations auxquelles l'élève doit se soumettre, en lien avec le respect de l'assiduité et dans le prolongement des travaux écrits exigés. La participation orale est valorisée par les enseignants car elle vient étayer l'avancée du cours. Ce sont en effet les enseignants qui en sont les maîtres d'œuvre: ils en fixent les règles et l'organisent. Cette participation orale fait d'ailleurs souvent l'objet d'une évaluation régulière pour laquelle chaque enseignant impose des critères qu'il a lui-même préalablement établis.

Des dispositifs pédagogiques mis en œuvre dès la rentrée 2000, comme les travaux personnels encadrés (TPE) et l'éducation civique, juridique et sociale (ECJS) apportent aux lycéens de nouvelles modalités d'expression, le premier par l'autonomie relative accordée aux élèves dans la réalisation du projet, le second par l'introduction du débat à visée argumentative (Billouet, 2007).

Hormis ces formes de participation proposées dans le cadre de la classe, les élèves ont la possibilité de participer à d'autres activités, à caractère associatif ou dont l'objet apparaît plus politique, organisées cette fois en dehors des heures de cours.

Si la vie associative (foyer socio-éducatif, union nationale des sports scolaires, etc.) est plutôt considérée favorablement de la part des adultes, perçue comme un temps de détente, un espace de rencontres et d'échanges, une ouverture culturelle, la participation aux instances politiques du lycée, depuis le mandat du délégué de classe jusqu'à celui de membre du conseil d'administration, est nettement plus problématique.

Plusieurs points sont ici à relever, en particulier la nette distinction entre instances consultatives et instances délibératives. Tant que l'avis des élèves n'est que consultatif, les oppositions adultes se font peu sentir car l'enjeu reste modeste: il s'agit surtout d'écouter les remarques, éventuellement les doléances des élèves et d'accepter d'y être attentif. Par contre, quand l'avis formulé a une portée décisionnelle, délibérative, certains adultes de l'établissement craignent ne pouvoir maîtriser les débats et rétablir si besoin la situation en leur faveur (Condette, 2007). Lorsque l'on observe le déroulement du conseil d'administration, on constate clairement que les possibilités d'expression accordées aux élèves lors de ces réunions formelles sont rapidement limitées, circonscrites, cadrées par un rituel qui vient dérouter les élèves non initiés, étouffant alors toute volonté participative. C'est un monde que les élèves élus qualifient volontiers « d'étrange », de « complexe», dans lequel ils disent ne pas vraiment se sentir à l'aise. Dans une certaine mesure, on peut en dire autant du fonctionnement du conseil de classe, instance dans laquelle les élèves délégués sont invités à prendre brièvement la parole pour évoquer quelques considérations générales qui, de toute 
façon, ne remettront pas en cause l'organisation interne de la classe et le travail des enseignants. C'est d'ailleurs la consigne que donnent généralement le chef d'établissement et/ou le professeur principal, en tout début de conseil de classe:

"Au préalable, je précise toujours aux personnes présentes, aux élèves délégués mais aussi à l'ensemble des autres membres du conseil, et ceci, vous comprenez, pour éviter toute forme de dérapage... je dis donc qu'il ne peut y avoir une remise en cause de qui que ce soit pendant le conseil... N'oublions pas que nous sommes là pour juger le travail des élèves, il ne faut donc pas s'y tromper ${ }^{4}$."

On touche ici au point névralgique sur lequel se cristallisent toutes les discussions au sujet du bien-fondé de la participation des élèves. Si l'on se réfère aux textes officiels, on peut lire que les élèves délégués ont la possibilité de s'exprimer sur toutes les questions portant sur la vie de la classe. Pourtant, dans les faits, les choses en vont autrement car la vie de la classe ne peut inclure les relations élèves/ enseignants et doit nécessairement se limiter au travail fourni par les élèves, à leur comportement, aux éventuelles perturbations, avec parfois le droit d'intervenir sur l'élaboration du planning des devoirs surveillés ou sur la répartition des devoirs à effectuer à la maison. Autrement dit, la vie de la classe ne peut être abordée que sous l'angle du travail et de l'évaluation de l'élève, l'enseignant et sa pédagogie étant formellement exclus de ce processus.

La thématique de la participation revient ainsi à s'interroger sur la véritable part de pouvoir que détiennent les élèves dans l'établissement. La question du pouvoir, et en particulier la question du pouvoir des usagers de l'école (parents et élèves), apparaît en effet comme un enjeu majeur dans les débats internes qui animent la vie de l'établissement.

\section{La participation des élèves et la question du partage du pouvoir dans l'établissement}

La demande de participation à l'organisation et au fonctionnement de l'établissement peut alors venir faire irruption dans un monde relativement fermé et ordonné qui souvent se méfie du regard extérieur et rechigne à la fois à rendre des comptes aux usagers de l'école, et à envisager le changement ou l'évolution des normes établies depuis déjà un certain nombre d'années, des normes stables et partagées par une grande partie de la communauté des personnels.

La participation des élèves, notamment des délégués, introduit ainsi un bouleversement possible dans l'organisation scolaire. Si les collégiens ont tendance à s'autolimiter quand ils sentent que leur parole est accueillie avec une certaine froideur, quand ce n'est pas de l'hostilité, les lycéens - et c'est ce qui fait leur spécificité - sont plutôt disposés à défendre leur point de vue, même s'ils estiment que cela peut éventuellement leur porter préjudice. L'organisation scolaire semble davantage reposer au lycée sur un ordre négocié, et il devient difficile pour les

4. Extrait d'un entretien avec le proviseur d'un LEGT. 
personnels d'imposer une décision sans la moindre concertation, sans la moindre explication, ou il faut s'attendre à des réactions d'opposition, parfois vives, de la part des élèves. Celles-ci peuvent aller de la réclamation individuelle (pour un devoir qui n'aurait pas été « justement » évalué) jusqu'à des perturbations graves, collectives, parfois anonymes, s'accompagnant alors souvent dans ce dernier cas de dégradations et se prolongeant en dehors de la classe.

Ces élèves, parfois avec violence, se réclament d'un principe de justice (Kellerhals, 2008), s'appuyant sur des points précis du règlement intérieur qui n'auraient pas été appliqués ou sur une interprétation subjective de certaines règles. En tout cas, ils demandent réparation, estimant que la parole leur a été confisquée et qu'ils sont victimes d'un préjudice.

Le développement de ces formes de juridicisation dans l'espace scolaire interroge le fonctionnement de l'école (Toulemonde, 2006). Cela signifie en effet que les règles de droit doivent impérativement remplacer le droit coutumier construit sur des habitudes, des « traditions » propres à un établissement, et par conséquent il n'est plus impossible pour les personnels de devoir rendre des comptes, d'apporter des justifications si elles sont demandées. Les textes officiels de juillet $2000^{5}$ relatifs pour les deux premiers aux punitions et sanctions dans l'établissement scolaire et pour le troisième à l'élaboration et l'application du règlement intérieur vont d'ailleurs tout à fait dans ce sens et ne manquent pas de créer des tensions, d'autant que les élèves et leur famille ont une bien meilleure connaissance de leurs droits et qu'ils entendent s'en servir si besoin. Si ces textes permettent de limiter les éventuels abus de pouvoir de la part des personnels, ils les obligent également à être prudents: « penser juridiquement devra devenir un réflexe ${ }^{6}$. »

Dans cette perspective, la relation inégalitaire traditionnellement non discutée et non discutable tend quelque peu à se transformer, notamment entre enseignants et élèves. L'autorité ne va plus de soi, même pour des professeurs expérimentés ne rencontrant en principe pas de problèmes de discipline, mais devient au contraire une quête voire une conquête permanente. Elle est sans cesse à reconstruire ou à rééquilibrer, ce qui n'est pas sans déstabiliser nombre d'adultes, en particulier les enseignants qui voient là une atteinte à l'exercice de leur activité professionnelle, et même, pour certains, une atteinte à leur statut d'enseignant. Certains, à ce sujet, incriminent les pratiques démocratiques qui se sont développées en faveur des élèves, ces dernières années:

"Je crois qu’on est allé trop loin... parce qu’on a laissé croire aux élèves qu’ils pouvaient discuter de tout, d'une manière complètement égalitaire; c'est une grave erreur; et à présent on en subit les conséquences ${ }^{7}$. "

5. Décret n 2000-620 du 5 juillet 2000 relatif aux établissements publics locaux d'enseignement; Décret n 2000-633 du 6 juillet 2000 relatif aux procédures disciplinaires dans les collèges, les lycées et les établissements d'éducation spéciale; circulaire 2000-106 relative au règlement intérieur dans les EPLE

6. Buttner Y., Maurin A., Thouveny B., Le droit dans la vie scolaire. Dalloz, 4édition, 2007.

7. Extrait d'un entretien avec un enseignant de lycée. 
La participation effective des élèves peut apparaître, au quotidien, comme un puissant perturbateur des relations entre élèves et adultes.

\section{Des regards divergents sur la participation des élèves}

Si une partie des enseignants, et parfois aussi des chefs d'établissement, s'interroge sur la réelle mise en œuvre, voire le bien-fondé, de la participation des élèves, les conseillers principaux d'éducation semblent porter un regard sensiblement différent.

\section{Le statut professionnel et les missions inhérentes à la fonction exercée}

Les chefs d'établissement, même s'ils se déclarent dans l'ensemble plutôt favorables à la participation des lycéens, gardent souvent tout de même une certaine méfiance à l'égard des nouveaux droits accordés. La difficulté majeure, selon eux, réside dans le fait de "savoir canaliser toutes ces énergies »: "Il faut éviter tout dérapage, j'ai encore à l'esprit les dernières manifestations [lycéennes]. Notre responsabilité est aussi de protéger les élèves. " (entretien avec un proviseur). Un autre chef d'établissement déclare: "Si on leur laisse vraiment la parole, il est fort à penser qu'ils ne nous la rendront plus... ou alors difficilement, après des négociations à n'en plus finir! » La crainte vient ici du fait que ces nouveaux droits, en particulier le droit d'expression, peuvent s'exercer au plus haut niveau (comme au conseil d'administration) et sont susceptibles de perturber le fonctionnement interne de l'établissement basé sur une faible remise en cause des décisions des adultes, et par conséquent de mettre à mal la réputation du lycée fondée essentiellement sur des critères de performance scolaire et de respect des règles. Et l'on sait que les revendications et manifestations lycéennes sont souvent perçues dans l'opinion comme des formes d'inscription de la jeunesse dans l'espace public, pour reprendre les propos d'A. Vulbeau ${ }^{8}$, mais elles sont aussi souvent interprétées comme le signe, pour l'établissement (et a fortiori pour celui qui le dirige), d'une faible capacité à garantir l'ordre ou à le rétablir rapidement.

Pour leur part, les conseillers principaux d'éducation (CPE) encouragent les élèves à participer aux différentes instances institutionnelles, l'objectif visé étant de les inciter à prendre des initiatives dans la vie lycéenne, mais aussi de contribuer à former de futurs citoyens responsables et actifs dans la société. Les missions qui définissent leur fonction les y invitent fortement ${ }^{9}$. En effet, l'animation éducative, troisième champ d'activité professionnelle, porte sur la responsabilisation des élèves à travers la préparation des diverses élections des délégués, l'accompa-

8. Vulbeau A., Les inscriptions de la jeunesse. L'Harmattan, 2002.

9. Circulaire 82-482 relative au rôle et aux conditions d'exercice de la fonction des conseillers d'éducation et des conseillers principaux d'éducation. 
gnement et la formation de ces élèves mandatés par leurs pairs. Les missions des CPE répondent donc tout à fait aux demandes officielles formulées dans divers décrets ou circulaires relatifs à la vie scolaire et à la participation des élèves.

Mais les conditions d'exercice de ces missions facilitent sans conteste la prise en compte effective de l'implication des élèves. En effet, le CPE ne fait pas cours, et à ce titre, il a une certaine latitude dans l'organisation de son travail, et peut inclure assez facilement dans un emploi du temps qui n'est pas figé, des séances de formation des délégués, des réunions d'élèves, des heures de vie de classe portant sur des questions de vie scolaire... En outre, sa relation aux élèves diffère considérablement de celle de l'enseignant. Le CPE a rarement en face de lui et pour une durée relativement longue des groupes d'élèves. Il les rencontre plutôt de manière ponctuelle, en fonction des informations qu'il a à leur transmettre ou des conseils qu'il souhaite leur prodiguer. Son statut au final l'expose moins que celui de l'enseignant.

S'ils sont pour la plupart favorables aux instances participatives, les CPE se disent néanmoins limités dans leur champ d'action par les autres adultes de l'établissement et en particulier les enseignants qui, selon leurs propos, «ne travaillent pas dans le même sens ». Ils regrettent notamment le peu d'intérêt et d'implication de ces personnels lors des séances de formation des délégués qu'ils organisent.

Les enseignants semblent en effet assez partagés sur la question de la participation des élèves. Certains se déclarent favorables à la valorisation de la parole de l'élève parce qu'ils défendent " des valeurs et des pratiques de citoyenneté " (entretien avec un enseignant de LEGT); "c'est la démocratie qui est en jeu », affirme un enseignant de lycée professionnel. D'autres, au contraire, ne voient pas l'intérêt de ces nouveaux droits et de leur mise en ouvre ("c'est une réforme de plus... »). Deux raisons majeures peuvent expliquer cette méfiance ou ce refus: les enseignants peuvent craindre de « se laisser déborder » car l'animation de groupes requiert des capacités qu'ils ne possèdent pas forcément; ils disent d'ailleurs qu'« ils n'ont pas été formés pour cela »; mais surtout ils souhaitent, selon les propos qu'ils utilisent, "préserver l'essentiel », c'est-à-dire mettre l'accent sur l'apprentissage des savoirs disciplinaires, d'autant que, régulièrement, les cours sont interrompus, perturbés par un certain nombre de tâches ou d'interventions qui "n'ont rien à voir avec l'enseignement et qui font perdre beaucoup de temps »: distribution ou ramassage de documents administratifs, informations à donner oralement ou à faire écrire dans le carnet de correspondance, intervention de différents collègues (assistants d'éducation, infirmière scolaire, conseiller d'orientation psychologue, CPE, etc.). Les enseignants, pour une grande part, considèrent que la seule participation " utile et efficace pour les élèves », pour reprendre leurs propos, reste la participation en classe car elle est, selon eux, un adjuvant à la réussite scolaire.

En outre, les professeurs, en conformité avec leur statut, sont constamment placés devant des groupes d'élèves. Cela explique que tous ne partagent pas cette conception d'un élève actif, futur citoyen, car certains craignent que l'élève 
actif ne devienne trop réactif. C'est sans doute pour cette raison qu'ils préfèrent cantonner le jeune dans un rôle d'élève placé sous contrôle, ne lui accordant alors que de maigres marges de manœuvre.

Pourtant le statut de l'enseignant et les missions qu'il doit exercer incluent la prise en compte de cette dimension participative : la circulaire de $1997^{10}$ précise en effet les missions des enseignants, indiquant explicitement qu'elles ne peuvent se limiter à la transmission d'un savoir mais doivent aussi initier les élèves à la prise de responsabilités au sein de l'établissement. C'est en partie le message que la formation générale professionnelle tente de diffuser.

\section{La formation des personnels}

La formation initiale des personnels consiste encore pour le moment en une alternance entre un stage en responsabilité effectué dans un collège ou un lycée et des séances de formation disciplinaire et générale organisées dans un institut universitaire de formation des maîtres (IUFM), désormais école interne d'une université.

La formation générale professionnelle, parce qu'elle réunit des personnels d'enseignement et d'éducation (enseignants de diverses disciplines, conseillers principaux d'éducation, professeurs documentalistes), propose une approche transversale des questions d'éducation, et permet la confrontation et l'enrichissement des pratiques de chacun. La connaissance de l'établissement, l'apprentissage des règles de vie, la gestion des incidents et des conflits, la complémentarité professionnelle entre les divers personnels, la connaissance et la reconnaissance du statut de l'élève, l'éducation à la citoyenneté sont autant de thématiques abordées lors de ces séances, qui permettent de faire émerger des idées communes, mais aussi des points de divergence que l'ensemble des membres du groupe en formation tente d'identifier et d'analyser. Les méthodes pédagogiques basées sur l'utilisation de techniques de groupes, en lien avec les nouveaux dispositifs d'enseignement (éducation civique juridique et sociale, travaux personnels encadrés, projets pluridisciplinaires à caractère professionnel...) sont, là encore, autant de thématiques étudiées qui visent à développer et promouvoir des espaces de participation destinés à conduire l'élève vers une plus grande autonomie dans les apprentissages et dans les choix personnels et professionnels qu'il aura à réaliser.

Il n'en reste pas moins que les efforts entrepris en formation sont à prolonger, et même sans doute à accentuer car les clivages professionnels et les cloisonnements disciplinaires sont encore nombreux. Malgré une explicitation des divers statuts professionnels s'appuyant sur les textes officiels, malgré un travail commun sur les pratiques de chacun, on remarque que les personnels enseignant et d'éducation fonctionnent en formation initiale sur des représentations souvent erronées

10. Circulaire n 97-123 du 23 mai 1997 relative à la mission du professeur exerçant en collège, lycée d'enseignement général et technologique ou en lycée professionnel. 
qui renvoient à leur expérience personnelle, une expérience parfois pas très ancienne... de leur propre parcours d'élève, et dont ils ont du mal à se détacher. Il y a donc fort à faire pour dépasser ce stade des représentations et faire entrer chacun dans la construction d'une professionnalité que l'on sait évolutive, et qui s'enrichira progressivement des réussites mais aussi des échecs (Rey, 1999). Apprendre à travailler autrement, développer des compétences transversales pour " préparer les élèves à affronter la complexité du monde » (Perrenoud, 2003), tel est le défi à relever. Dans cette perspective, il s'agit d'accepter de prendre des risques, le risque de se confronter à l'altérité, le risque de se tromper dans ses choix, le risque de partager ses doutes, le risque de faire confiance aux élèves et aussi, et ce n'est pas le moindre... le risque de se faire confiance, c'est-à-dire d'apprendre à mieux se connaître, dans ses convictions, ses capacités, mais aussi dans ses limites, pour engager un processus de réflexion et d'analyse qui permettra sinon de lever définitivement les doutes, du moins de les mettre à distance, de les transformer en outils réflexifs (Inisan, 2004). Engagés dans ce processus de formation ou de transformation, les personnels d'enseignement et d'éducation pourront assumer ce que Meirieu nomme « la dimension politique » d'un métier qui utilise l'acte d'enseigner comme vecteur d'éducation à la citoyenneté.

\section{Conclusion}

La thématique de la participation lycéenne permet d'éclairer des conceptions différentes de l'élève et d'éventuels a priori sur l'exercice de ses droits. Si l'implication dans des activités associatives ou directement liées au fonctionnement de la classe ne semble pas poser problème, il n'en va pas de même dès lors que l'on s'intéresse aux possibilités et modalités de participation à l'organisation de l'établissement. La question du partage du pouvoir émerge alors inévitablement et génère des débats qui peuvent se montrer constructifs mais elle fait aussi et surtout apparaître des divergences entre des personnels qui pourtant travaillent ensemble en établissement et sont censés poursuivre les mêmes buts. À travers le statut et la pratique professionnelle, on voit se dessiner différents profils d'éducateurs, certains privilégiant la responsabilisation de l'élève, d'autres se montrant plus réticents face à des jeunes qui pourraient remettre en cause leur autorité. En cela, la formation professionnelle est un levier utile pour appréhender de manière collective les difficultés inhérentes à l'ensemble des métiers de l'éducation et tenter d'y apporter non pas des recettes, mais des outils pour que chacun puisse se construire progressivement une professionnalité qui ne soit pas incompatible avec l'implication et la responsabilisation des jeunes.

Sylvie Condette-Castelain, laboratoire CIREL-Profeor (EA 4354), université Charles-de-Gaulle, Lille 3. 


\section{BIBLIOGRAPHIE}

AUdiGIER François. L'éducation à la citoyenneté. INRP, 1999.

Billouet Pierre (coord.), Débattre. Pratiques scolaires et démarches éducatives. L'Harmattan, 2007.

CONDETTE Sylvie. Participer à la vie du lycée: entre propositions formelles et réalisations effectives, La revue de la FOEVEN, 2007, n 152 La parole des jeunes, p. 7-13.

CONDETTE Sylvie. La participation des lycéens à la vie de leur établissement. Lille, ANRT, 2005.

CONDETte-CAstelain Sylvie. La participation lycéenne et le rapport des élèves à la loi, Spirale, n 34, octobre 2004, p. 75-91.

ESTABlet Roger. (coord), Radiographie du peuple lycéen. ESF, 2005.

GALICHET François. L'école, lieu de citoyenneté. ESF, 2005.

Henaff Gaël, Merle Pierre. (dir.), Le droit et l'école. De la règle aux pratiques. Rennes, Presses universitaires de Rennes, 2003.

Inisan Jean-François (coord.). Analyses de pratiques et attitudes réflexives en formation, Actes de l'école d'été des IUFM du pôle nord-est, Reims, CRDP de Champagne-Ardenne \& SCEREN, 2004.

Kellerhals Jean, LANGUin Noëlle. Juste? Injuste? Sentiments et critères de justice dans la vie quotidienne, Payot, 2008.

Mabilon-Bonfils Béatrice, SaAdoun Laurent. Sociologie politique de lécole. PUF, 2001.

MeIrIEU Philippe. Lettre à un jeune professeur. ESF, 2005.

Paquay Léopold, Altet Marguerite, Charlier Évelyne, Perrenoud Philippe. (dir.), Former des enseignants professionnels. Quelles stratégies? Quelles compétences? Bruxelles, De Boeck, 1996.

Perrenoud Philippe. L'école est-elle encore le creuset de la démocratie? Lyon, Chronique sociale, 2003.

ReMY Régis (dir.), Conseiller principal d’éducation. Regards sur le métier. Paris, Adapt éditions, 2007. REY Bernard, Les relations dans la classe, au collège et au lycée. ESF, 1999.

TOULEMONDE Bernard. Droit et responsabilité dans l'institution scolaire, in Bernard Toulemonde (dir.) Le système éducatif en France. La Documentation française-CNED, 2006, p. 20-25.

XYPAS Constantin (dir.). Les citoyennetés scolaires. De la maternelle au lycée. PUF, 2003.

\section{ANNEXE I}

\section{Indications méthodologiques}

L'article proposé s'appuie sur plusieurs types de données:

- Une partie des résultats produits et analysés dans le cadre d'une recherche doctorale réalisée de 1999 à 2003 dans deux lycées d'enseignement général et technologique de l'académie de Lille. Ces résultats sont complétés depuis 2004 par des enquêtes plus ciblées dans d'autres lycées.

Le choix de ces terrains d'enquête a été guidé par un double critère: la promotion de la participation lycéenne à la fois proposée officiellement comme l'un des axes du projet d'établissement, et mise en œuvre concrètement au quotidien. 
Les données empiriques ont été recueillies lors d'observations nombreuses (en privilégiant l'observation participante sur des périodes scolaires longues) et d'entretiens semi-directifs menés auprès d'un échantillon d'environ 140 élèves et des personnels des différents lieux d'accueil.

- Les textes officiels relatifs aux missions des enseignants et des conseillers principaux d'éducation. Nous nous appuyons également sur les contenus de la formation disciplinaire des CPE et sur les contenus de la formation générale professionnelle (formations dispensées à l'université d'Artois-IUFM Nord Pas-de-Calais).

- 8 entretiens semi-directifs menés auprès de CPE ( 2 en formation initiale, 3 en collège, 3 en LP et LEGT) de septembre à novembre 2006; 10 entretiens semidirectifs menés auprès d'enseignants en 2005-2006 (2 en formation initiale, 3 en LP, 5 en LEGT); 6 entretiens avec des chefs d'établissements de février 2005 à octobre 2006 ( 2 en collège; 2 en LP; 2 en LEGT).

Les entretiens, d'une durée d'une heure environ, s'appuyaient sur quelques questions guides: 1. Que signifie pour vous la vie lycéenne? 2. En quoi consistet-elle dans votre établissement? (variante pour le collège: est-ce que, en prévision de l'entrée au lycée, vous travaillez cette question avec vos collégiens?). 3. Quels en sont les objectifs? 4. Quel sens y donnez-vous? 5. Est-ce que vous y prenez part (si oui, de quelle façon)? 